\title{
AVALIAÇÃO DE ANOREXÍGENOS E SUAS ASSOCIAÇÕES PRESCRITAS EM UMA FARMÁCIA COM MANIPULAÇÃO DO MUNICÍPIO DE TOLEDO-PR.
}

\author{
EVALUATION OF ANORECTIC AND ASSOCIATIONS PRESCRIBED IN A MAGIS- \\ TRAL PHARMACY OF TOLEDO'S CITY, PARANÁ.
}

\author{
ANDREA BEJOLA ${ }^{1}$, SIMONE MARIA MENEGATTI DE OLIVEIRA ${ }^{2}$, SUZANE VIR- \\ TUOSO2.
}

${ }^{1}$ Discente de Especialização em Farmacologia Aplicada da UNIOESTE - PR

${ }^{2}$ Docente do Curso de Farmácia da UNIOESTE - PR

Email: simone_meneoli@yahoo.com.br

\begin{abstract}
RESUMO
A obesidade é uma enfermidade crônica com repercussões negativas para a saúde, e sendo considerada, na sociedade moderna, como uma epidemia global. Este índice vem aumentando devido à ingestão excessiva de calorias e um gasto insuficiente de energia associados com um estilo de vida sedentário. Este trabalho sobre o consumo de anorexígenos apresenta um perfil parcial, mas atual, sobre a farmacoterapia da obesidade, tomando como base as prescrições aviadas em uma farmácia magistral. Tem por objetivo avaliar as prescrições de medicamentos anorexígenos sujeitos a controle especial em uma farmácia de manipulação do município de Toledo - PR, verificando quais são os mais prescritos, suas associações, posologia, especialidade do médico prescritor e sexo do usuário do medicamento. Com os resultados encontrados, concluiu-se que ainda são prescritas associações com fármacos anorexígenos pouco estudadas ou danosas ao usuário, apesar da coibição legal, e que problemas quanto ao correto preenchimento da receita ainda necessitam ser sanados.
\end{abstract}

Palavras-chave: formulações farmacêuticas, farmacoterapia na obesidade, anorexígenos

\begin{abstract}
Obesity is a chronic disease with repercussions for the health, and being considered, in modern society, as a global epidemic. This index has been increasing due to excessive intake of calories and a lack of energy expenditure associated with a sedentary way of life. This work about of the consumption of anorectics presents a partial profile, but current, about the pharmacotherapy of obesity, based on prescriptions dispensed in a magistral pharmacy. Its purpose is to assess the prescriptions of anorectic drugs subject to special control in a magistral pharmacy of Toledo's city - PR, verifying what are the most frequently prescribed, their associations, dosage, doctor's specialty and sex of the drug user with the results found, it was concluded that are still prescribed the associations with anorectic drugs little studied or harmful to the user, despite the legal restraint, and that problems with the correct completion of prescription still need to be remedied.
\end{abstract}

Key words: pharmaceutical formulations, pharmacotherapy in obesity, anorectics

\section{1 . INTRODUÇÃO}

A obesidade é uma doença crônica, multifatorial, e atualmente representa um dos maiores desafios de saúde pública em todo o mundo. À medida que se consegue erradicar a miséria entre as camadas mais pobres da população brasileira, a obesidade 
desponta como um problema mais freqüente e mais grave que a desnutrição (CORREA, 2005). Os medicamentos anorexígenos são muito prescritos pela classe médica atualmente (GUY-GRAND,1987). O alto índice de prescrição destes medicamentos deve-se também ao aumento do percentual de obesos em nosso país. (CORREAA, 2005).

O consumo de estimulantes psicomotores constituídos pelas anfetaminas e seus derivados encontra-se atualmente entre os mais importantes problemas de saúde. O uso continuado e em doses excessivas poderia levar à degeneração das células cerebrais, podendo ocorrer lesões irreversíveis (UNESP, 2003), apesar disso, a anfetamina é uma das drogas que mais ganha usuários a cada ano. No Brasil, a ONU vem constantemente alertando sobre o crescimento do consumo das mesmas (ANVISA, 2006).

Muitas vezes os anorexígenos são prescritos em associação com outros fárfármacos na mesma formulação, como ansiolíticos, diuréticos, laxantes, hormônios, entre outros. $\mathrm{O}$ uso isolado ou combinado de diurético e laxantes não é apropriada para o tratamento de obesidade (ANVISA, 2006). O emprego irracional de diuréticos e laxantes durante período prolongado, podem promover danos á saúde, como a poliúria, desidratação, hipopocalemia, desequilíbrio hidroeletrolítico, arritmia cardíaca. Irritação intestinal dor abdominal danos ao cólon (BEHAR, 2002). A prescrição de levotiroxina, triodotironina e Triac $®$ (tiratricol) para perda de peso é ineficaz na promoção de perda de tecido adiposo e, na verdade, acentua a perda de tecido magro, provocando em equilíbrio nitrogenado negativo. Existe também o risco de cardiotoxidade em indivíduos susceptíveis e a possibilidade de se provocar hipertiroidismo (ANFARMAG, 2006).

As associações de medicamentos para o controle de peso não são bem estudadas, e não apresentam eficácia e segurança estabelecidas, sendo que estas terapias combinadas envolvendo a utilização de mais de um fármaco para obesidade são contra-indicadas até que haja mais informações disponíveis a respeito da eficácia e segurança em tratamento em longo prazo envolvendo a associações destes fármacos. Por esta razão, a Portaria do Ministério da Saúde número 344 de doze de maio de mil novecentos e noventa e oito, assim como os Conselhos Federais de Medicina e de Farmácia nas orientações para seus profissionais, condenam a prescrição simultânea de drogas tipo anfetaminas com outros fármacos de uso controlado, como benzodiazepínicos, diuréticos, hormônios ou extratos hormonais e laxantes (ANVISA, 2006).

Os efeitos ansiogênicos da Marapuama são comparáveis ao do pentilenetetrazol. Quando a Marapuama foi avaliada no teste de estereotipia induzida por anfetaminas em camundongos albinos, os animais apresentaram convulsão, cianose e morte. $O$ mesmo aconteceu quando a Marapuama foi testada quanto à proteção da letalidade induzida por anfetamina em camundongos. Esta combinação, associada à espirulina, hidroclorotiazida e cáscara sagrada, é uma das mais encontradas nas formulações ditas naturais para emagrecer (TEIXEIRA, 2003).

Os benzodiazepínicos estão entre os medicamentos mais usados no mundo todo, havendo estimativas de que entre 1 e $3 \%$ de toda a população ocidental já os tenha consumido regularmente por mais de um ano (BALDESSARINI, 1995; HUF, 2000). Entre os antidepressivos, Teixeira (2003) relata que os inibidores de captação de serotonina têm sido mais freqüentemente utilizados, por serem mais seguros e mais 
bem tolerados. A fluoxetina é atualmente o medicamento antidepressivo mais prescrito no Brasil e no mundo, havendo indícios de que possa atuar na promoção de perda de peso durante vários meses após o início da terapia.

Diante do exposto, este trabalho teve como objetivo avaliar as prescrições de medicamentos anorexígenos de controle especial em uma farmácia de manipulação do município de Toledo-PR, identificando os mais prescritos, as associações de outros fármacos com os anorexígenos, sua posologia, a especialidade do médico prescritor, o preenchimento dos dados da receita e o sexo do paciente.

\section{MATERIAL E MÉTODOS}

O estudo foi desenvolvido por meio de uma análise quantitativa de 900 receitas e notificações de receitas de medicamentos anorexígenos sujeitos a controle especial conforme Portaria SVS/MS n 344 de 12 de maio de 1998 e retidas em uma farmácia com manipulação do município de Toledo - PR, no período referente aos meses de 01 de janeiro a 31 de outubro de 2006.

Os itens analisados foram: nome dos anorexígenos prescritos, doses, associação com outros fármacos em formulações a serem administradas no mesmo horário, a especialidade do profissional prescritor, além da verificação da prescrição como um todo, verificando a forma de prescrição das quantidades, da forma farmacêutica, presença ou ausência do preenchimento das informações referentes ao paciente/ comprador/ prescritor, incorreção dos dados da gráfica e dispensação do medicamento fora do prazo previsto na legislação.

\section{RESULTADOS E DISCUSSAO}

Dos receituários analisados, constatou-se que somente em $50 \%$ (450) deles os anorexígenos são prescritos como monodroga. Os fármacos mais encontrados foram o femproporex, representando $26 \%$ (232) do total e a anfepramona $12 \%$ (109). Nos últimos anos, um fármaco que vem ganhando espaço é a sibutramina, encontrada em $11 \%$ (99) das receitas. Além destes, o mazindol com $0.5 \%$ (05) e a selegilina, com $0.5 \%$ (05), foram também verificados (Figura 01).

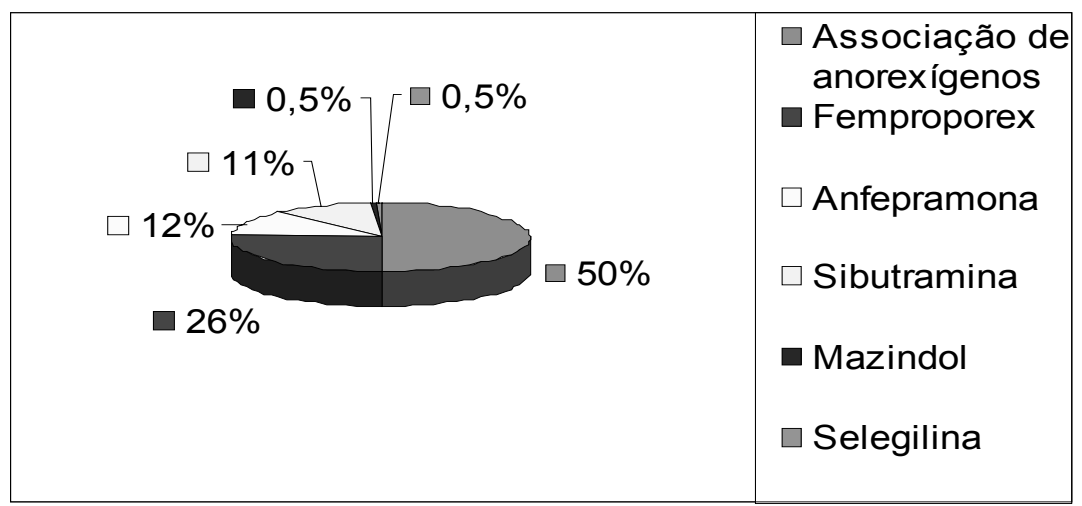

FIGURA 1. MEDICAMENTOS ANOREXÍGENOS PRESCRITOS EM ASSOCIAÇÃO E COMO MONODROGA 
Tais quantidades são confirmadas por NOTO et al (2002), que relata em seu artigo que, quanto aos supressores de apetite, a maior porcentagem de notificações $(95,0 \%)$ foi encontrada nas farmácias com manipulação e sugerem que as mesmas são a maior fonte de dispensação destas substâncias. Os supressores de apetite mais prescritos foram a anfepramona e o femproporex.

Os anorexígenos prescritos associados a outros fármacos foram o femproporex, em 30\% (270) dos casos e a anfepramona encontrada em 20\% (180) das receitas. A sibutramina, manzidol e selegilina não foram encontrados com associações.

Das receitas de anorexígenos prescritas em associação com outros fármacos (50\%), 31\% (279) das mesmas contêm a associação de vários fitoterápicos na mesma receita, sendo eles a aloína, a cavalinha, a centella asiática e a passiflora. Em $8 \%(72)$ das receitas, os anorexígenos encontram-se associados ao sene, e em 5\% (45), à marapuama. Quanto às associações com fármacos ansiolíticos, destaca-se o bromazepam, encontrado em 5\% (45) das receitas e o diazepam, em 1\% (09) das mesmas (Figura 2).

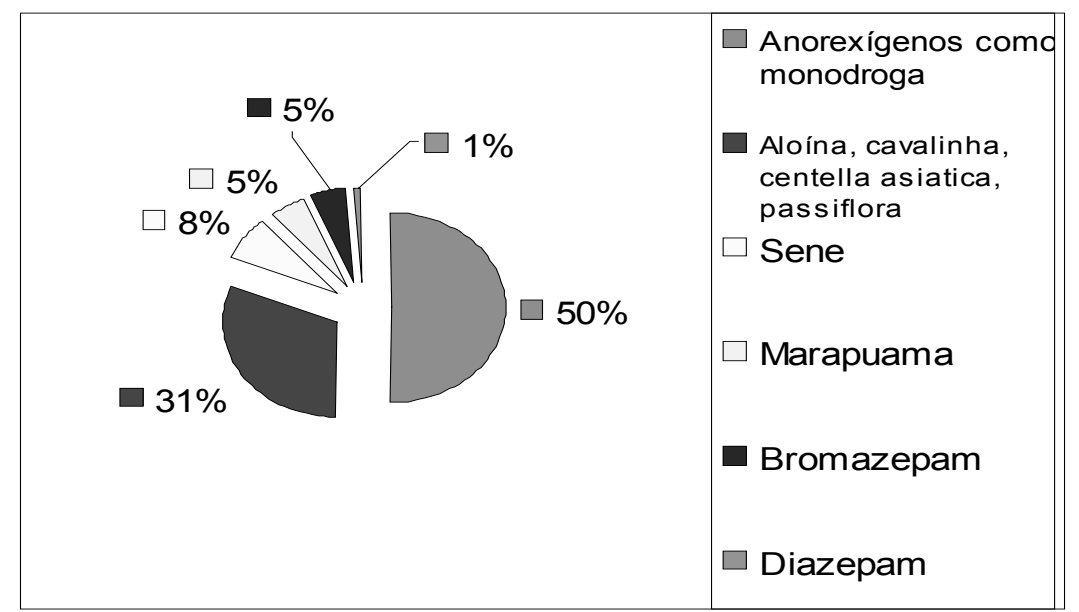

FIGURA 2. FORMULAÇÕES CONTENDO ANOREXÍGENOS ASSOCIADOS A OUTROS FÁRMACOS

Quanto às associações de fitoterápicos destacadas acima, não foi encontrado nenhum estudo científico comprovando a eficácia do uso simultâneo destas drogas.

Neste trabalho ficou evidenciado que grande parte dos anorexígenos são prescritos acompanhados de um ansiolítico e/ou de um composto fitoterápico, sendo estes prescritos em receitas separadas, mas com administração simultânea. A prescrição destes fármacos na mesma receita é proibida pela Portaria 344/98 (ANVISA, 1998).

Quanto à relação entre a especialidade do prescritor e o perfil de prescrição, pôde-se observar que as especialidades são muito variáveis, e em 15\% (135) delas não foi possível identificar a mesma, por falta do carimbo ou identificação do prescritor. Das prescrições com especialidade identificada (85\%), a maioria foi emitida por endocrinologistas, representando 35\% (315) das mesmas. Em seguida, os clínicos gerais com $28 \%$ (252), os ginecologistas com $20 \%$ (180), seguidos de $2 \%$ (22) de cardiologistas (Figura 3). 


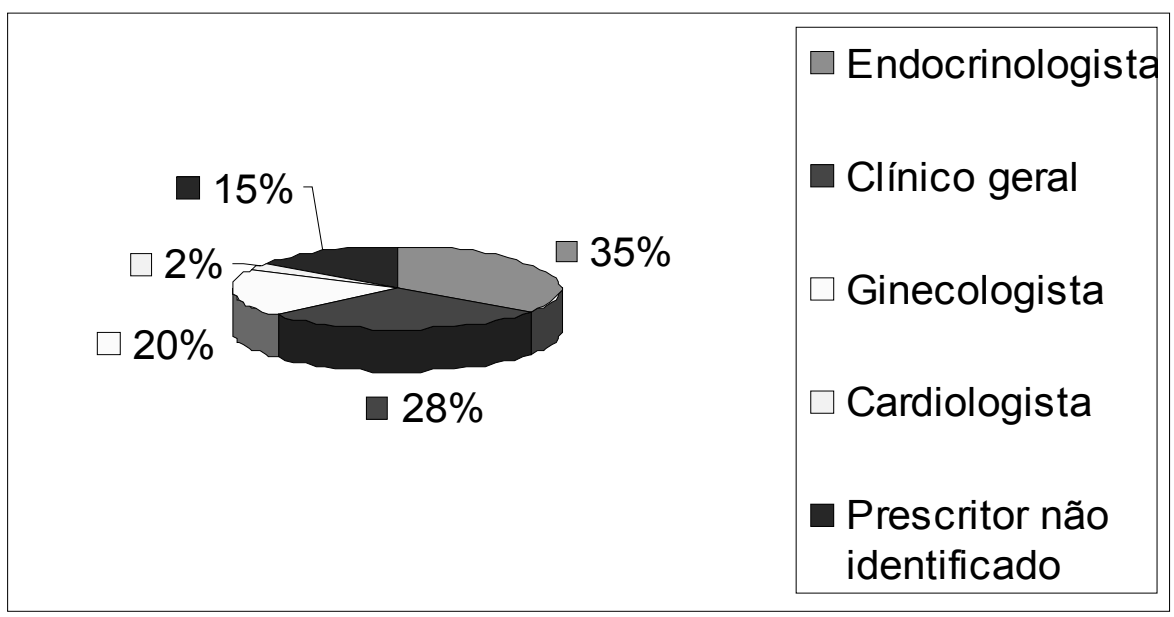

FIGURA 3. PRESCRIÇÃO POR ESPECIALIDADE MÉDICA

As prescrições efetuadas pelos endocrinologistas foram encontradas em maior número, contudo, seguida de perto pelos clínicos gerais. Tais dados permitem supor que mesmo sendo o uso de anorexígenos feito sob orientação médica, a utilização dos mesmos pode estar ocorrendo de maneira inadequada, pois as literaturas especializadas recomendam que a prescrição de tais fármacos para casos específicos de tratamento de redução de peso deve ser conduzida por endocrinologista ou outros especialistas do assunto (ANVISA, 2006).

Quanto à quantidade do medicamento ser apresentada em número arábico, como prevê a legislação, observou-se o correto preenchimento em $97 \%$ (873) das receitas. No restante das mesmas, $1 \%$ (09) não havia o número arábico e em 2\% (18) o mesmo estava ilegível.

A quantidade prescrita em extenso estava presente somente em $11 \%$ (95) das receitas. A posologia estava ausente em $6 \%$ (55).

A dosagem e forma farmacêutica foram descritas em $99 \%$ (890) das mesmas. Com relação à dosagem preconizada pela literatura, 98\% (882) das receitas estavam de acordo com a mesma, sendo que $2 \%$ (18) estavam acima da dosagem.

Quanto às falhas no preenchimento das notificações/receitas citadas acima, o resultado observado é preocupante, visto que o não preenchimento correto dos dados da substância prescrita a ser manipulada, pode levar a dificuldades no processo de manipulação, assim como na informação terapêutica, ou orientação farmacêutica a ser prestada ao paciente.

Quanto à presença ou ausência do preenchimento das informações referentes ao paciente/comprador nas notificações/receitas, a ausência do endereço do paciente é a falha mais freqüente, verificados em $50 \%$ (450) das receitas. É importante ressaltar que a localização do paciente/comprador é fundamental para que se possa evitar a possibilidade de fraudes na prescrição/dispensação, e considerando a legislação vigente, tal situação pode configurar tráfico de entorpecentes.

Também foram verificados problemas de preenchimento nas notificações/receitas quanto à ausência de dados do prescritor em 15\% (135) das mesmas, e na dispensação do medicamento fora do prazo previsto na legislação, fato observado em $15 \%$ 
(135) dos receituários. Estes erros são considerados graves e indicam a necessidade de intensificação dos processos de fiscalização sanitária.

Quanto ao sexo dos usuários desta classe de medicamento, constatou-se que as mulheres adquiriram $80 \%$ (720) dos mesmos, enquanto os homens consumiram $20 \%(180)$.

\section{CONSIDERAÇÕES FINAIS}

Este trabalho revelou um alto consumo de psicotrópicos anorexígenos na população feminina estudada, e sugere uma forte conexão entre o uso desta classe de medicamentos com a busca por padrões estéticos que a sociedade preconiza, onde a magreza é essencial nos dias de hoje.

O estudo demonstrou que somente metade dos anorexígenos prescrita foi encontrada como monodroga. A outra metade estava associada a algum tipo de fitoterápico e/ou ansiolítico. Apesar das campanhas atuais por parte dos órgãos competentes e da legislação ser muito clara no sentido de coibir o uso simultâneo destes fármacos, ainda se constata a não conscientização por parte dos prescritores, ao indicar associações carentes de maiores estudos científicos que comprovem sua segurança e eficácia.

Os resultados também evidenciam um cumprimento parcial da legislação referente a medicamentos de regime de controle especial, tanto por parte dos prescritores, quanto das farmácias.

O quadro atual de prescrição e consumo de anorexígenos sugere uma melhoria na qualidade da fiscalização governamental sobre os profissionais relacionados a estes medicamentos, mas também de uma melhor capacitação e conscientização dos mesmos, garantindo ao usuário uma real solução para seu problema de saúde, eliminando este ciclo vicioso que em nada colabora para a melhoria da saúde pública.

\section{REFERÊNCIAS}

Associação Nacional de farmacêuticos magistrais. Informações básicas para Prescrição e aviamentos racionais e seguros. Disponível em: <http//www.anfarmag.org. br> Acesso em 30 set 2006

BALDESSARINI, R.J. Drugs and the treatment of psychiatric disorders:

psychosis and anxiety. In: HARDMAN, J.G.; GILMAN, A.G.; LIMBIRD, L.E., Eds. Goodman \& Gilman's the pharmacological basis of therapeutics. 9 ed. New York: McGraw Hill, 1995. Cap. 18, p. 399 - 430.

BRASIL.Agência Nacional de Vigilância Sanitária. Portaria N³44, de 12 de maio de 1998. Dispõe sobre o regulamento técnico sobre substâncias e medicamentos sujeitos a controle especial. Brasília, 1998.

BRASIL. Agência Nacional de Vigilância Sanitária. Alerta sobre riscos no uso de 
anorexígenos Brasília, 2006. Disponível em: <http//www.anvisa.gov.br/divulga/noticias/ 2005/>. Acesso em 31 ago 2006.

BEHAR.R.Anorexigenos:Indicaciones e Interacciones. Rev.Chile. Neuro.Psiquiatr, 2002. n2. p-21-36.

CORREAA, LÍVIA. Avaliação do efeito da sibutramina sobre a saciedade por escala visual analógica em adolescentes obesos . Arq Bras Endocrinol Metab, Abr 2005, vol.49, no.2, p.286-290.

GUY-GRAND, B.L. Term Pharmacotherapy in the mangement of obsety. In. Bjorntorp.Rossner S. Eds. From Theory to Practice: Obsity in Europe: 88 London:John Libbey, 1989:311-318.

HUF G.; LOPES C.S.; ROSENFELD, S. O uso prolongado de benzodiazepínicos em mulheres de um centro de convivência para idosos. Cad. Saúde Pública, Rio de Janeiro, v. 16, n. 2, p. 351-62, 2000.

NOTO, A.R.; CARLINI, E.A.; MASTROIANNI, P.C.; ALVES, V.C.; GALDUROZ, J.C.; KUROIWA, W. ; CSIZMAR, J.; COSTA, A.; FARIA, M.A.; HIDALGO, S.R.; ASSIS, D.; NAPPO, S.A. Análise da prescrição e dispensação de medicamentos psicotrópicos em dois municípios do Estado de São Paulo. Rev. Bras. Psiquiatr., São Paulo, v. 24, n. 2, p. 68-73, 2002.

TEIXEIRA, L. Riscos da associação de marapuama com anorexígenos em fórmulas para emagrecer. Arq. Brás. Endocrinol. Metab., Out 2003, vol.47, no.5, p.632-632. 